\title{
Objectively measured physical activity and sedentary time in young adults born preterm-The ESTER study
}

\author{
Marjaana Tikanmäki ${ }^{1,2}$, Tuija Tammelin ${ }^{3}$, Nina Kaseva', Marika Sipola-Leppänen ${ }^{1,2,4}$, Hanna-Maria Matinolli, ${ }^{1,2}$, Harto Hakonen ${ }^{3}$, \\ Ulf Ekelund ${ }^{5}$, Johan G. Eriksson 1,6,7, Marjo-Riitta Järvelinn ${ }^{8,9,10,11}$, Marja Vääräsmäki ${ }^{4,12}$ and Eero Kajantie ${ }^{1,4,13}$
}

BACKGROUND: Young adults born preterm have higher levels of cardio metabolic risk factors and they report less physical activity than their peers born at term. Physical activity provides important cardio metabolic health benefits. We hypothesized that objectively measured physical activity levels are lower and time spent sedentary is higher among preterm-born individuals compared with controls.

METHODS: We studied unimpaired participants of the ESTER birth cohort study at age 23.3 y (SD: 1.2): 60 born early preterm (<34wk), 108 late preterm (34-36wk), and 178 at term (controls). Physical activity and sedentary time were measured by hip-worn accelerometer (ActiGraph).

RESULTS: As compared with controls' (mean physical activity, 303 counts per minute (cpm; SD 129)), physical activity was similar among adults born early preterm (mean difference $=$ $21 \mathrm{cpm}, 95 \% \mathrm{Cl}-61,19)$ or late preterm $(5 \mathrm{cpm},-27,38)$. Time spent sedentary was also similar. Adjustments for early life confounders or current mediating characteristics did not change the results.

CONCLUSION: In contrast to our hypothesis, we found no difference in objectively measured physical activity or time spent sedentary between adults born preterm and at term. The previously reported differences may be limited to physical activity captured by self-report.

$\mathrm{F}$ ollow-up studies of adolescents and adults born preterm suggest that those born smallest report less physical activity and are less fit than term-born individuals (1-4). The differences can be substantial: as assessed by a comprehensive 12-mo physical activity questionnaire, unimpaired adults born preterm at very low birth weight $(<1,500 \mathrm{~g})$ report over $50 \%$ lower energy expenditure from leisure-time conditioning physical activity than controls born at term (3). This may contribute to the increased cardio metabolic risk profile reported in this group $(5,6)$. However, these infants constitute only a minority of preterm infants; for example, in the United States, $70 \%$ of preterm infants are born late preterm, between 34 and 36 wk of gestation (7). Recent studies have suggested that the adverse cardio metabolic risk profile and perhaps levels of physical activity, and in parallel muscular fitness, decrease with an increasing degree of prematurity and may extend to those born late preterm $(8,9)$.

Promoting increased physical activity and decreased sedentary time is important for preventing cardio metabolic risk $(10,11)$. However, few studies have measured physical activity and sedentary time objectively in adults born preterm (12-14). We hypothesized that objectively measured physical activity levels are lower and sedentary time higher among pretermborn individuals and that there is a dose-response relationship between the degree of prematurity and physical activity and sedentary time.

\section{METHODS \\ Participants}

The participants come from the ESTER (Ennenaikainen syntymä ja aikuisiän terveys (Preterm Birth and Early-Life Programming of Adult Health and Disease) ) Preterm Birth Study comprising 1980 young adults. They were identified through the Northern Finland Birth Cohort 1986 (NFBC; 49.8\%; born in 1985-1986) or the Finnish Medical Birth Register (FMBR; 50.2\%; born in 1987-1989 in the same geographical area). In 2009-2011,753 individuals with verified length of gestation participated in a clinical study at 23.3 (SD 1.23) y (8). All participants were offered an accelerometer, if available.

After exclusions (described in Figure 1), 60 participants born early preterm (below $34 \mathrm{wk}$ of gestation), 108 born late preterm (between 34 and $36 \mathrm{wk}$ ), and 178 controls born after 37 completed weeks of gestation (referred to as "at term") were unimpaired, and had sufficient accelerometer data (Figure 1).

\section{Ethics}

The study was approved by the Coordinating Ethics Committee at Helsinki and Uusimaa Hospital District. All participants provided written informed consent.

\footnotetext{
'Department of Health, Chronic Disease Prevention Unit, National Institute for Health and Welfare, Oulu and Helsinki, Finland; ${ }^{2}$ Institute of Health Sciences, University of Oulu, Oulu, Finland; ${ }^{3}$ LIKES Research Center for Physical Activity and Health, Jyväskylä, Finland; ${ }^{4}$ PEDEGO Research Unit (Research Unit for Pediatrics, Dermatology, Clinical Genetics, Obstetrics and Gynecology), Medical Research Center Oulu (MRC Oulu), Oulu University Hospital and University of Oulu, Oulu, Finland; ${ }^{5}$ Department of Sport Medicine, Norwegian School of Sport Sciences, Oslo, Norway; ${ }^{\circ}$ Department of General Practice and Primary Health Care, University of Helsinki and Helsinki University Hospital, Helsinki, Finland; ; ${ }^{7}$ olkhälsan Research Center, Helsinki, Finland; ${ }^{8}$ Department of Epidemiology and Biostatistics, MRC-PHE Centre for Environment \& Health, School of Public Health, Imperial College London, United Kingdom; ${ }^{9}$ Faculty of Medicine, Center for Life Course Epidemiology, University of Oulu, Oulu, Finland; ${ }^{10}$ Biocenter Oulu, Oulu, Finland; ${ }^{11}$ Unit of Primary Care, Oulu University Hospital, Oulu, Finland; ${ }^{12}$ Department of Welfare, Children, Adolescents and Families Unit, National Institute for Health and Welfare, Oulu, Finland; ${ }^{13}$ Children's Hospital, Helsinki University Hospital, University of Helsinki, Helsinki, Finland. Correspondence: Marjaana Tikanmäki (marjaana.tikanmaki@thl.fi) 


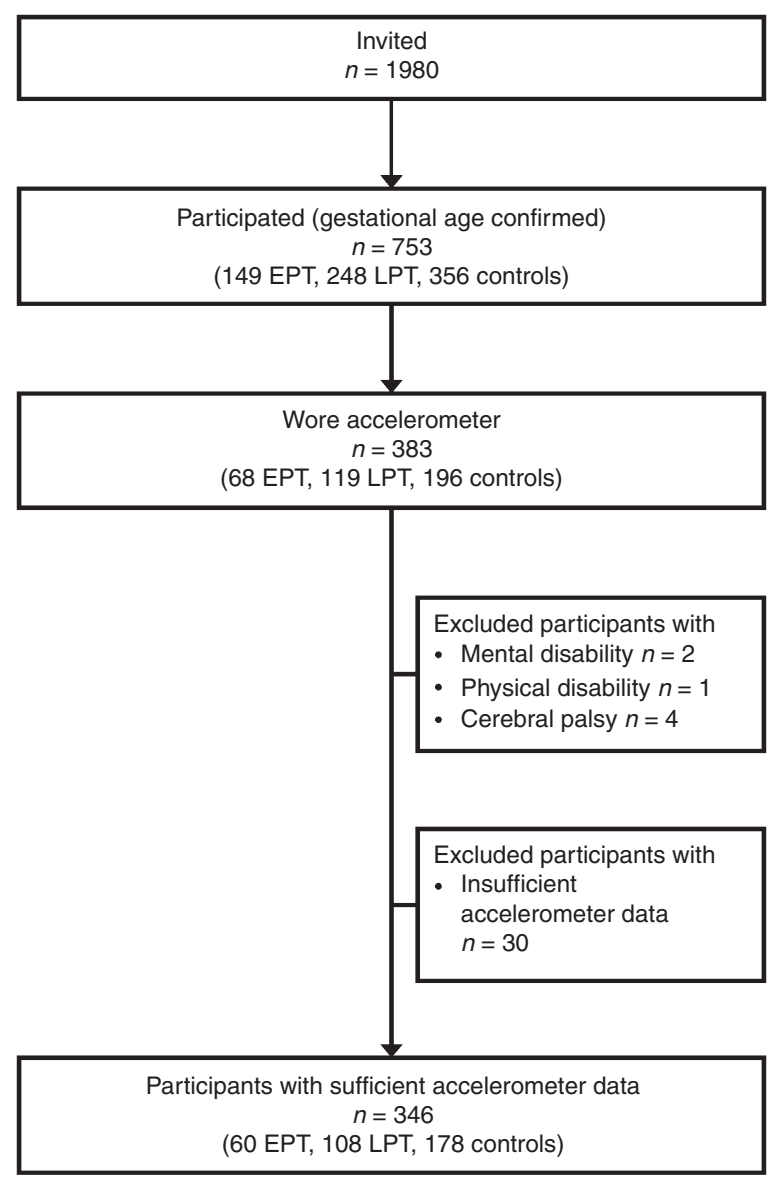

Figure 1. Flow chart of the study population. EPT, early preterm $(<34$ gestational wk); LPT, late preterm (34-36 gestational wk).

\section{Perinatal Data}

Perinatal data for participants recruited through the Northern Finland Birth Cohort 1986 comes from the cohort database, originally collected from hospital and maternal welfare clinic records (15). We collected corresponding data for those invited through the Finnish Medical Birth Register. Length of gestation (16) and maternal gestational diabetes, hypertension (gestational or chronic), or pre-eclampsia (including superimposed) diagnoses according to prevailing criteria were independently confirmed by reviewing original hospital records $(17,18)$. Small for gestational age (SGA) was defined as birth-weight SD score $<-2$ SD according to Finnish standards (19).

\section{Clinical Examination}

Clinical measurements included anthropometry. Body composition was assessed by using segmental multifrequency bioelectrical impedance (InBody 3.0, Biospace, Seoul, Korea). Muscular fitness was measured with the number of modified push-ups performed in $40 \mathrm{~s}$ and maximal handgrip strength of the dominant hand $(\mathrm{N})$ and cardiorespiratory fitness with heart rate at the end of a 4-min step test (9). Data on medical history, socioeconomic status, daily smoking, and self-reported physical activity (9), were collected with questionnaires. Childhood socioeconomic status was assessed as the educational attainment of the more highly educated parent enquired at the time of clinical examination.

\section{Physical Activity and Sedentary Time}

Physical activity was measured with an accelerometer (ActiGraph GT1M, ActiGraph, Pensacola, Florida) worn on the right hip during waking hours for seven consecutive days (20). Participants with valid data for at least 500 min per d on 2 weekdays and 1 weekend day were included (20). The epoch length was $60 \mathrm{~s}$, and nonwearing time was defined as continuous zero activity for $>60 \mathrm{~min}$. The outcomes were overall physical activity (counts per minute, $\mathrm{cpm}$ ), sedentary time (\%/d, <100 cpm), light-intensity physical activity (LPA; min/d, 100$1,951 \mathrm{cpm}$ ), moderate-to-vigorous intensity physical activity (MVPA; $\mathrm{min} / \mathrm{d}, \geq 1,952 \mathrm{cpm}$ ), and $\mathrm{MVPA}_{10 \mathrm{~min}}(\mathrm{~min} / \mathrm{d})$ that was defined as continuous MVPA lasting at least $10 \mathrm{~min}$ at a time $(21,22)$, with a 1 -min interruption allowed within a 5-min time frame. Accelerometerbased sedentary time was expressed as a proportion of daily monitoring time, thus, participants who wore the accelerometers for different lengths of time per day were comparable. Self-reported physical activity was calculated in MET hours per week (MET = metabolic equivalents; ratio of metabolic rate during exercise and estimated metabolic rate at rest; one MET corresponds energy expenditure of $\sim 1 \mathrm{kcal} / \mathrm{kg}$ $\times \mathrm{h}$ ) on the basis of a questionnaire on (i) light (assuming a value of three METs), (ii) moderate to vigorous (five METs), and (iii) commuting (four METs) physical activity (9).

\section{Statistical Methods}

We examined sex adjusted correlations between outcome variables and self-reported physical activity or physical fitness with Pearson's partial correlation. We compared the characteristics of the early and late preterm groups with those of the controls by using Student's $t$-test and the $\chi^{2}$-test, with Yeates' Correction for Continuity for 2 by 2 tables, and the outcomes using linear regression with a significance level of $P<0.05$. We tested for interactions between early and late preterm birth, and sex (significance level of $P<0.01$ ) by including a product term with these variables. Categorical adjusting variables were entered as dummy variables with separate dummy indicating missing values. In Model 1 we adjusted for sex, age, cohort, and the season of physical activity measurement (December-February, March-May, June-August, and September-November). In Model 2 we adjusted for potential early life confounders: parental education, maternal gestational diabetes, gestational hypertension, and birth weight SD score. In Model 3, we adjusted for potential adult mediators: asthma (23), height $(2,3)$, body fat percentage (8), and smoking (24). Because of skewed distribution, we also reran the analyses after $\log$-transforming $(\log 10($ variable +1$))$ the outcome variables to attain normality, adjusting for SGA status, and replacing the adjustment for body fat percentage with lean body mass.

A detailed nonparticipant analysis of the ESTER Preterm Birth Study has been described previously (8). Among the participants of the clinical study, we now compared the participants of the accelerometer study with the nonparticipants comprising those who did not attend accelerometer registration or were excluded from the analyses for insufficient accelerometer data (Figure 1). In this comparison, there was no significant difference in the participation of early or late preterm individuals as compared with the controls. As to participant characteristics listed in Table 1, the nonparticipants born preterm or at term were more likely to smoke daily $(P<0.001-0.026)$. The women of the control group participated more actively than men $(P=$ $0.044)$, and they had higher body mass index $(P=0.007)$ and body fat percentage $(P=0.004)$ as compared with the nonparticipants. Among early preterm nonparticipants cardiorespiratory fitness was slightly lower $(P=0.006)$. We performed analyses using SPSS 22.0 (IBM SPSS Statistics, IBM, Armonk, NY).

\section{RESULTS}

Participant characteristics are presented in Table 1 and outcomes by exposure group in Table 2. Sex adjusted correlation coefficient between objectively measured daily physical activity (cpm) and self-reported physical activity was 0.25 , and between objectively measured physical activity and fitness measures correlation coefficients were -0.29 for cardiorespiratory fitness and 0.12 for the number of modified push-ups performed. Other correlations between objectively measured physical activity and self-reported physical activity or fitness measures were similar or less. The mean physical 


\section{Articles | Tikanmäki et al.}

Table 1. Perinatal, neonatal, and current characteristics of young adults born preterm and their controls born at term

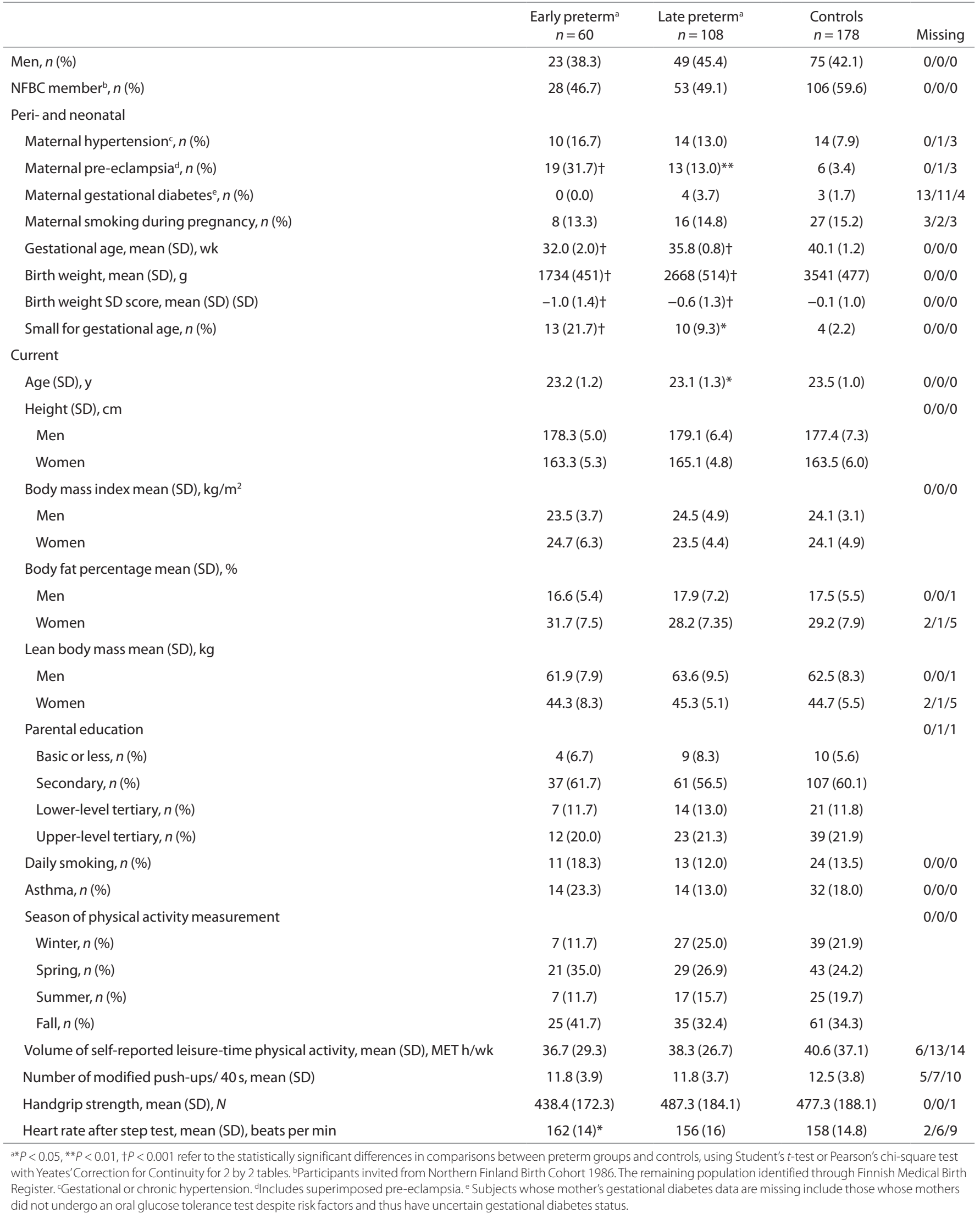


Table 2. Mean physical activity and sedentary time values of young adults born preterm and their controls born at term

\begin{tabular}{|c|c|c|c|}
\hline & $\begin{array}{l}\text { Early preterm } \\
\qquad n=60\end{array}$ & $\begin{array}{l}\text { Late preterm } \\
\quad n=108\end{array}$ & $\begin{array}{c}\text { Controls } \\
n=178\end{array}$ \\
\hline \multicolumn{4}{|c|}{ Time of wearing accelerometer, mean (SD), $\mathrm{min} / \mathrm{d}^{\mathrm{b}}$} \\
\hline Men & $859(73)$ & $886(90)$ & $892(83)$ \\
\hline Women & $869(62)$ & $881(79)$ & $870(84)$ \\
\hline All & $865(66)$ & $883(84)$ & $879(84)$ \\
\hline \multicolumn{4}{|c|}{ Daily physical activity, mean (SD), counts per minute } \\
\hline Men & $263(105)$ & $341(191)$ & $315(114)$ \\
\hline Women & $293(102)$ & $283(118)$ & $294(138)$ \\
\hline All & $282(103)$ & $309(157)$ & $303(129)$ \\
\hline \multicolumn{4}{|c|}{ Sedentary time, mean (SD), \% of daily monitoring time ${ }^{b}$} \\
\hline Men & $68.2(9.3)$ & $63.9(11.8)$ & $64.5(10.8)$ \\
\hline Women & $64.4(7.9)$ & $65.8(9.1)$ & $64.5(8.3)$ \\
\hline All & $65.9(8.6)$ & $65.0(10.4)$ & $64.5(9.4)$ \\
\hline \multicolumn{4}{|c|}{ Light-intensity physical activity, mean (SD), min/ $\mathrm{d}^{\mathrm{b}}$} \\
\hline Men & $247.5(83.0)$ & $280.9(97.6)$ & $283.2(103.5)$ \\
\hline Women & $281.5(74.1)$ & $272.3(83.0)$ & $280.5(77.2)$ \\
\hline All & $268.5(78.7)$ & $276.2(89.6)$ & $281.7(88.9)$ \\
\hline \multicolumn{4}{|c|}{ MVPA, mean (SD), $\mathrm{min} / \mathrm{d}^{\mathrm{b}}$} \\
\hline Men & $28.6(18.5)$ & $38.2(30.4)$ & $35.0(20.6)$ \\
\hline Women & $28.4(16.9)$ & $29.2(19.5)$ & $29.7(21.0)$ \\
\hline All & $28.5(17.4)$ & $33.3(25.3)$ & $31.9(20.9)$ \\
\hline \multicolumn{4}{|c|}{$\mathrm{MVPA}_{10 \min }(\mathrm{SD}), \mathrm{min} / \mathrm{d}^{\mathrm{b}}$} \\
\hline Men & $7.5(9.3)$ & $9.8(13.5)$ & $8.5(11.9)$ \\
\hline Women & $13.0(13.6)$ & $11.8(12.3)$ & $12.0(14.2)$ \\
\hline All & $10.9(12.4)$ & $10.9(12.8)$ & $10.6(13.3)$ \\
\hline
\end{tabular}

a $P$-values refer to comparisons between preterm groups and controls, using Student's t-test or Pearson's chi-square test with Yeates' Correction for Continuity for 2 by 2 tables. Level for statistical significance $P$-value $<0.05$. ${ }^{\text {}}$ The variables were computed as weighted averages of daily physical activity (PA) during weekdays (WD) and weekend days (WED) (daily PA - ( $5 *$ average WD PA + $2 *$ average WED PA) / 7).

activity level and sedentary time were similar among young adults born early and late preterm compared with the controls. Adjustments for potential early life confounders or current mediating characteristics did not change the results (Table 3 ). The results remained when log-transformed outcome variables were used and when adjusted for SGA and lean body mass instead of body fat percentage and when we excluded pregnant individuals. There was no interaction between association of sex and preterm birth with the outcomes.

\section{DISCUSSION}

We objectively measured physical activity and sedentary time with a hip-worn accelerometer in young adults born early and late preterm, as compared with their peers who were born at term. In contrast to our hypothesis, we did not observe any difference in physical activity levels or the proportion of sedentary time per day between young healthy adults born early or late preterm and controls born at term. Accounting for a wide range of early life confounders and possible adult mediators did not change our results. Although our sample size did not allow us to exclude subtle differences, our findings suggest preterm birth is not a determinant of physical activity captured by accelerometry in adulthood.

Our findings contradict earlier observations of lower selfreported physical activity among adults born preterm (2-4). This discrepancy parallels previous observations among young very-low-birth-weight adults who reported undertaking $\sim 50 \%$ less conditioning physical activity than controls (2-4). These findings were not replicated when physical activity was assessed objectively $(12,13)$. Self-report and accelerometry reflect different aspects of physical activity. Self-report enables the assessment of a broad range of physical activities in any circumstances providing an average of physical activity in a longer period of time. Objective measurement is again more precise in registering the intensity of physical activity and sedentary time in a shorter time frame (25). The correlations between accelerometer-measured and self-reported physical activity tend to be low-to-moderate, in this study 0.25 and in recent meta-analyses around 0.3-0.4 (25-27). Indeed, both types of measurements have been reported to be associated with cardio metabolic and other health benefits $(11,28-31)$, although associations with accelerometer-measured physical activity tend to be stronger (31). These methodological differences may explain why very low birth weight adults report lower levels of conditioning leisure-time physical activity (3) and lower sports participation (2) despite this has not been detected with objective measurement in the same cohort (32) or in our present study including the full range of preterm birth.

\section{Strengths and Limitations}

The strengths of our study include objective measurement of physical activity and sedentary time with a reliable hip-worn accelerometer with a 7-d-registration (20) and a study population enabling the evaluation of physical activity across the full range of preterm birth. We also had access to reliable and diverse perinatal data, including verified length of gestation. It is possible that individuals who are more physically active may have participated in the accelerometry study. The lower rate of smoking among participants would seem to suggest this (33) as also in our study smoking was associated with lower amount of continuous moderate-to-vigorous physical activity lasting at least $10 \mathrm{~min}$ at a time and self-reported physical activity. However, fitness levels were similar in participants and nonparticipants, except among early preterm nonparticipants cardiorespiratory fitness was slightly lower than among early preterm participants. The potential participation of more active individuals would only be expected to introduce bias if the association between preterm birth and physical activity would be different among participants and nonparticipants. This seems unlikely although cannot be excluded. 


\section{Articles $\mid$ Tikanmäkietal.}

Table 3. Daily physical activity (mean counts per minute, cpm), sedentary time (\% of monitoring time), light physical activity (min/d), moderate to vigorous physical activity (MVPA, $\mathrm{min} / \mathrm{d})$ and $\mathrm{MVPA}_{10 \mathrm{~min}}(\mathrm{~min} / \mathrm{d})$ in preterm groups compared with term-born controls

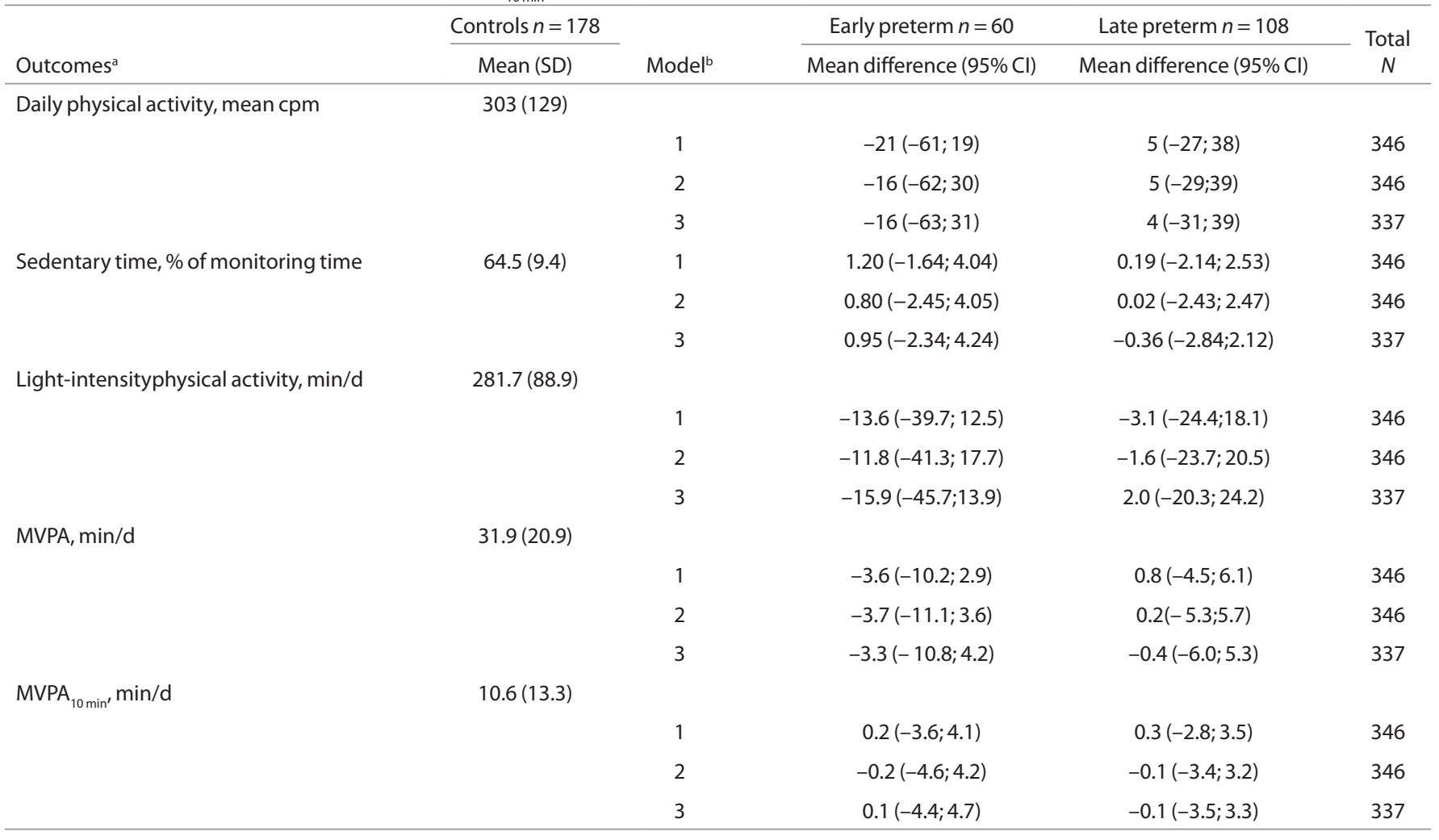

$\mathrm{Cl}$, confidence interval.

aThe variables were computed as weighted averages of daily physical activity (PA) during weekdays (WD) and weekend days (WED) (daily PA - $(5 *$ average WD PA + $2 *$ average WED PA) / 7). 'bodel 1: sex, age, cohort, season. Model 2: Model 1+ socioeconomic status, maternal smoking, gestational diabetes and hypertension, and birth weight SD score. Model 3: Model $2+$ diagnosed asthma, adult body size: height, body fat percentage, and smoking. No statistically significant $(P$-value $<0.01)$ interactions between sexes in the associations of preterm birth and the outcomes were observed.

\section{CONCLUSIONS}

In conclusion, we did not observe differences in objectively measured physical activity and sedentary time among young adults born early or late preterm compared with controls born at term. This suggests that lower levels of physical activity in adults born preterm are likely to be limited to physical activity preferentially captured by self-report, including broad range of activities that accelerometry is not able to detect in a short time frame.

\section{ACKNOWLEDGMENTS}

We are grateful to all the participants for their contribution in the ESTER Study. We also thank research nurses Katriina Inget, Sinikka Kursu, HannaMaria Matinolli, Liisa Saarikoski, Marjatta Takala, Sarianna Vaara, data manager Sigrid Rosten, research assistants Risto Karvonen, Petteri Koivurova, Antti Koskela, Sanna Mustaniemi, Heli-Kaisa Saarenpää, and Marja Vanhatalo from the National Institute of Health and Welfare, Oulu and Helsinki, Finland, as well as statistician Anna Kankaanpää from LIKES Research Center for Physical Activity and Health, Jyväskylä, Finland.

\section{STATEMENT OF FINANCIAL SUPPORT}

The ESTER Study was supported by grants from the Academy of Finland (SALVE program for 2009-2012 and grants 127437, 129306, 130326, 134791, and 263924 to EK), Doctoral Programme for Public Health, University of Tampere (to $\mathrm{MSL}$ ), the Emil Aaltonen Foundation (to EK), European Commission (Framework 5 award QLG1-CT-2000-001643; to MRJ), the Finnish Foundation for Pediatric Research (to EK), the Finnish Government Special Subsidiary for Health Sciences (evo) (to JGE), Finnish Medical Societies: Duodecim (to EK) and Finska Läkaresällskapet (to JGE and NK), the Jalmari and Rauha Ahokas Foundation (to EK), the Juho Vainio Foundation (to EK and MSL), the National Graduate School of Clinical Investigation (to MT), the Novo Nordisk Foundation (to EK and MV), the Signe and Ane Gyllenberg Foundation (to EK and JGE), the Sigrid Jusélius Foundation (to EK), and the Yrjö Jahnsson Foundation (EK, MSL, and MV). The supporters of the study had no role in the study design; the collection, analysis, and interpretation of data; the writing of the report; and the decision to submit the paper for publication.

Disclosure: There is no conflict of interest to disclose.

\section{REFERENCES}

1. Burns YR, Danks M, O'Callaghan MJ, et al. Motor coordination difficulties and physical fitness of extremely-low-birthweight children. Dev Med Child Neurol 2009;51:136-42.

2. Kajantie E, Strang-Karlsson S, Hovi P, et al. Adults born at very low birth weight exercise less than their peers born at term. J Pediatr 2010;157: 610-6, 616.e1.

3. Kaseva N, Wehkalampi K, Strang-Karlsson S, et al. Lower conditioning leisure-time physical activity in young adults born preterm at very low birth weight. PLoS One 2012;7:e32430.

4. Rogers M, Fay TB, Whitfield MF, Tomlinson J, Grunau RE. Aerobic capacity, strength, flexibility, and activity level in unimpaired extremely low birth weight $(<\mathrm{or}=800 \mathrm{~g})$ survivors at 17 years of age compared with term-born control subjects. Pediatrics 2005;116:e58-65.

5. Johansson S, Iliadou A, Bergvall N, Tuvemo T, Norman M, Cnattingius S. Risk of high blood pressure among young men increases with the degree of immaturity at birth. Circulation 2005;112:3430-6.

6. Kajantie E, Hovi P. Is very preterm birth a risk factor for adult cardiometabolic disease? Semin Fetal Neonatal Med 2014;19:112-7. 
7. Martin JA, Osterman MJ; Centers for Disease Control and Prevention (CDC). Preterm births - United States, 2006 and 2010. MMWR Suppl 2013;62:136-8

8. Sipola-Leppänen $M$, Vääräsmäki $M$, Tikanmäki $M$, et al. Cardiometabolic risk factors in young adults who were born preterm. Am J Epidemiol 2015;181:861-73.

9. Tikanmäki M, Tammelin T, Sipola-Leppänen $M$, et al. Physical fitness in young adults born preterm. Pediatrics 2016;137:1-10.

10. Hamilton MT, Healy GN, Dunstan DW, Zderic TW, Owen N. Too little exercise and too much sitting: inactivity physiology and the need for new recommendations on sedentary behavior. Curr Cardiovasc Risk Rep 2008;2:292-8.

11. Nocon M, Hiemann T, Müller-Riemenschneider F, Thalau F, Roll S, Willich SN. Association of physical activity with all-cause and cardiovascular mortality: a systematic review and meta-analysis. Eur J Cardiovasc Prev Rehabil 2008;15:239-46.

12. Kaseva N, Martikainen S, Tammelin T, et al. Objectively measured physical activity in young adults born preterm at very low birth weight. J Pediatr 2015;166:474-6.

13. Welsh L, Kirkby J, Lum S, et al.; EPICure Study Group. The EPICure study: maximal exercise and physical activity in school children born extremely preterm. Thorax 2010;65:165-72.

14. Hildebrand M, Kolle E, Hansen BH, et al. Association between birth weight and objectively measured sedentary time is mediated by central adiposity: data in 10,793 youth from the International Children's Accelerometry Database. Am J Clin Nutr 2015;101:983-90.

15. Järvelin MR, Hartikainen-Sorri AL, Rantakallio P. Labour induction policy in hospitals of different levels of specialisation. Br J Obstet Gynaecol 1993;100:310-5.

16. Sipola-Leppänen $M$, Vääräsmäki $M$, Tikanmäki $M$, et al. Cardiovascular risk factors in adolescents born preterm. Pediatrics 2014;134:e1072-81.

17. Miettola S, Hartikainen AL, Vääräsmäki M, et al. Offspring's blood pressure and metabolic phenotype after exposure to gestational hypertension in utero. Eur J Epidemiol 2013;28:87-98.

18. Vääräsmäki $M$, Pouta $A$, Elliot $\mathrm{P}$, et al. Adolescent manifestations of metabolic syndrome among children born to women with gestational diabetes in a general-population birth cohort. Am J Epidemiol 2009;169:1209-15.

19. Pihkala J, Hakala T, Voutilainen P, Raivio K. [Characteristic of recent fetal growth curves in Finland]. Duodecim 1989;105:1540-6.

20. Matthews CE, Ainsworth BE, Thompson RW, Bassett DR Jr. Sources of variance in daily physical activity levels as measured by an accelerometer. Med Sci Sports Exerc 2002;34:1376-81.
21. Haskell WL, Lee IM, Pate RR, et al. Physical activity and public health: updated recommendation for adults from the American College of Sports Medicine and the American Heart Association. Med Sci Sports Exerc 2007;39:1423-34.

22. Mutikainen S, Helander E, Pietilä J, Korhonen I, Kujala UM. Objectively measured physical activity in Finnish employees: a cross-sectional study. BMJ Open 2014;4:e005927.

23. Jaakkola JJ, Ahmed P, Ieromnimon A, et al. Preterm delivery and asthma: a systematic review and meta-analysis. J Allergy Clin Immunol 2006;118:823-30.

24. Strang-Karlsson S, Räikkönen K, Pesonen AK, et al. Very low birth weight and behavioral symptoms of attention deficit hyperactivity disorder in young adulthood: the Helsinki study of very-low-birth-weight adults. Am J Psychiatry 2008;165:1345-53.

25. Steene-Johannessen J, Anderssen SA, van der Ploeg HP, et al. Are selfreport measures able to define individuals as physically active or inactive? Med Sci Sports Exerc 2016;48:235-44.

26. Prince SA, Adamo KB, Hamel ME, Hardt J, Connor Gorber S, Tremblay M. A comparison of direct versus self-report measures for assessing physical activity in adults: a systematic review. Int J Behav Nutr Phys Act 2008;5:56.

27. Sofi F, Capalbo A, Cesari F, Abbate R, Gensini GF. Physical activity during leisure time and primary prevention of coronary heart disease: an updated meta-analysis of cohort studies. Eur J Cardiovasc Prev Rehabil 2008;15:247-57.

28. Chastin SF, Egerton T, Leask C, Stamatakis E. Meta-analysis of the relationship between breaks in sedentary behavior and cardiometabolic health. Obesity (Silver Spring) 2015;23:1800-10.

29. Ekelund U, Ward HA, Norat T, et al. Physical activity and all-cause mortality across levels of overall and abdominal adiposity in European men and women: the European Prospective Investigation into Cancer and Nutrition Study (EPIC). Am J Clin Nutr 2015;101:613-21.

30. Biswas A, Oh PI, Faulkner GE, et al. Sedentary time and its association with risk for disease incidence, mortality, and hospitalization in adults: a systematic review and meta-analysis. Ann Intern Med 2015;162:123-32.

31. Celis-Morales CA, Perez-Bravo F, Ibañez L, Salas C, Bailey ME, Gill JM. Objective vs. self-reported physical activity and sedentary time: effects of measurement method on relationships with risk biomarkers. PLoS One 2012; 7:e36345.

32. Kaseva N, Martikainen S, Tammelin T, et al. Objectively measured physical activity in young adults born preterm at very low birth weight. J Pediatr 2015;166:474-6.

33. Kaczynski AT, Manske SR, Mannell RC, Grewal K. Smoking and physical activity: a systematic review. Am J Health Behav 2008;32:93-110. 